\title{
Does Mechanical Threshold Inspiratory Muscle Training Promote Recovery and Improve Outcomes in Ventilator-Dependent ICU Patients? The IMPROVE Randomised Trial.
}

Bernie Bissett ( $\square$ Bernie.Bissett@canberra.edu.au )

University of Canberra https://orcid.org/0000-0002-3248-384X

I Anne Leditschke

Mater Hospital Brisbane

Teresa Neeman

Australian National University

Margot Green

Canberra Hospital

Vince Marzano

Australian National University

Katie Erwin

Canberra Hospital

Frank MP van Haren

Australian National University

\section{Robert Boots}

University of Queensland - Saint Lucia Campus: The University of Queensland

Jennifer Paratz

University of Queensland - Saint Lucia Campus: The University of Queensland

Research

Keywords: physiotherapy (techniques), breathing exercises, critical care, intensive care

Posted Date: August 31st, 2021

DOl: https://doi.org/10.21203/rs.3.rs-850486/v1

License: (c) (1) This work is licensed under a Creative Commons Attribution 4.0 International License.

Read Full License 


\section{Abstract}

\section{Background:}

In ventilator-dependent ICU patients, inspiratory muscle training may improve inspiratory muscle strength and accelerate liberation from the ventilator, but optimal training parameters are yet to be established and little is known about the impact of inspiratory muscle training on quality of life or dyspnea. Thus we sough to ascertain whether inspiratory muscle training, commenced while ventilator-dependent, would improve outcomes for ICU patients invasively ventilated for 7 days or longer.

\section{Methods:}

In this randomized trial with assessor-blinding and intention-to-treat analysis, 70 participants (mechanically ventilated $\geq 7$ days) were randomized to receive once- daily supervised high-intensity inspiratory muscle training with a mechanical threshold device in addition to usual care, or usual care (control). Primary outcomes were inspiratory muscle strength (maximum inspiratory pressure \% predicted) and endurance (fatigue resistance index) at ventilator-liberation and 1 week later, and quality of life (SF-36v2, EQ-5D). Secondary outcomes included dyspnea, physical function, duration of ventilation and in-hospital mortality.

\section{Results:}

33 participants were randomly allocated to the training group and 37 to control. There were no statistically significant differences in strength (maximum inspiratory pressure) (95\% $\mathrm{Cl}-7.4$ to 14.0 ) or endurance (fatigue resistance index) $(95 \% \mathrm{Cl}-0.003$ to 0.436$)$. Quality of life improved significantly more in the training group than control (EQ5D 17.2; 95\% Cl 1.3-33.0) (SF36-PCS 6.97; 96\% Cl 1.96-12.00). Only the training group demonstrated significant reductions in dyspnea (-1.5 at rest, -1.9 during exercise). There were no between-group differences in duration of ventilation or other measures. In-hospital mortality was higher in the control group than the training group ( 9 vs $4,24 \%$ vs $12 \%, p=0.23$ ).

\section{Conclusions:}

In ventilator-dependent patients, mechanical threshold loading inspiratory muscle training improves quality of life and dyspnea, even in the absence of strength improvements or acceleration of ventilator liberation.

Trial registration: ACTRN12610001089022

https://www.anzctr.org.au/Trial/Registration/TrialReview.aspx?ACTRN=12610001089022

Trial protocol: http://bmjopen.bmj.com/content/2/2/e000813.full

\section{Background}


Respiratory muscle rehabilitation is an important element of recovery for intensive care unit (ICU) patients who have experienced prolonged invasive mechanical ventilation(1). At the point of ventilator liberation, inspiratory muscle weakness is twice as prevalent as peripheral ICU-acquired weakness(2). A recent systematic review and meta-analysis concluded that inspiratory muscle training accelerates liberation from mechanical ventilation more so than conventional physical therapy(3). However there remains considerable heterogeneity in approaches to inspiratory muscle training in ICU patients $(1,4)$ and the ideal training parameters and techniques are yet to be established(1).

Mechanical spring-loaded threshold devices have been used to strengthen inspiratory muscles in patients recently weaned from mechanical ventilation. A supervised daily high-intensity strengthening regime (30 breaths at a minimum $50 \%$ maximum inspiratory pressure) improved ICU patients' inspiratory muscle strength and quality of life within 2 weeks(5). While this high-intensity approach to inspiratory muscle training is safe in selected ventilator-dependent ICU patients(6), it is not yet clear whether these patients would gain similar benefits from commencing training prior to ventilator liberation.

Previous studies of threshold-based inspiratory muscle training in ventilator-dependent patients have been limited by restrictive sampling (e.g. excluding patients younger than 70 or those with a tracheostomy(7); only targeting patients with $\operatorname{COPD}(8)$, or insufficient loading (e.g. $30 \%(9)$ or $40 \%(10)$ maximum inspiratory pressure). One recent randomized trial of high-intensity threshold-based inspiratory muscle training failed to detect improvements in inspiratory muscle strength or ventilation duration(11), however this study did not measure the effect of training on other patient-centred outcomes such as quality of life or dyspnea.

Thus, the objectives of our study were to establish if high-intensity inspiratory muscle training, using a mechanical threshold loading device, would improve patient-centred outcomes (including quality of life, dyspnea, and physical function) in a heterogeneous sample of ICU patients who were ventilatordependent for 7 days or longer.

\section{Methods}

\section{Study Design}

In accordance with our pre-published protocol(12), we conducted this investigator-initiated single-centre randomized controlled trial using concealed allocation, assessor-blinding and intention-to-treat analysis to compare inspiratory muscle training with usual care in ICU patients who were ventilator-dependent for at least 7 days. The study was approved by the Australian Capital Territory Health Human Research Ethics Committee (ETH.10.10.370) and the University of Queensland Medical Research Ethics Committee (2010001498). The published protocol(12) (trial registration ACTRN12610001089022) complied with the CONSORT guidelines for clinical trials(13).

Patients were eligible for inclusion if they had been invasively mechanically ventilated (via endotracheal tube or tracheostomy) for at least 7 days, were aged $\geq 16$ years, and were sufficiently alert to provide 
informed consent and participate actively in training (Riker Sedation-Agitation Scale(14) score of 4). Exclusion criteria included refusal to participate, pregnancy, significant pain or distress affecting breathing, medical instability or anticipated death within weeks. All participants provided written consent to participate in the study.

The study was conducted in a 31-bed Australian mixed medical/surgical/trauma ICU where minimal sedation and early rehabilitation(15) are well-established. The medical officers making ventilator liberation decisions were blinded to group allocation. Training was conducted by physiotherapists in line with our previously-published protocol, which is safe and feasible in ICU patients(6). Due to the nature of the supervised training, therapists could not be blinded to group allocation.

\section{Intervention}

Using a computer-generated random number sequence (with concealed allocation), participants were randomized to usual care (control group) or inspiratory muscle training in addition to usual care (IMT group). Usual care included secretion clearance techniques (e.g. percussion, hyperinflation and suction) but did not include inspiratory resisted breathing of any kind.

The IMT device used was the Threshold inspiratory muscle trainer (Threshold IMT device HS730, Respironics NJ, USA). This spring-loaded one-way valve provides titratable inspiratory resistance in a range of $9-41 \mathrm{cmH}_{2} \mathrm{O}$ and can readily be connected to an endotracheal tube or tracheostomy (Fig. 1).

For training, a high-intensity low-repetition method was used as previously described $(1,5,16)$. Intensity was prescribed at a minimum of $50 \%$ of maximal inspiratory pressure (MIP) at the highest tolerable intensity where the participant could just complete the 6th breath in a set of 6 breaths. One treatment session consisted of 5 sets of 6 breaths, where resistance was increased between sets as appropriate. Participants were returned to the ventilator between sets, where they typically required only a few minutes' rest.

Training commenced following randomisation and continued once daily (weekdays only) until 1 week following successful liberation from mechanical ventilation (defined as 24 hours without positive pressure). We did not use a sham device for comparison due to the risk of a sham device providing a training stimulus in participants with very low inspiratory muscle strength(17).

\section{Measures}

Primary outcomes were measured by specifically-trained research nurses blinded to group allocation. Initial measurements were conducted following enrolment and prior to randomisation; interim measurements were obtained following successful liberation from the ventilator ( 24 hours spontaneously breathing without positive pressure); and final measurements were recorded 1 week following liberation. Inspiratory muscle strength (MIP) was measured from residual volume using a portable MicroRPM Respiratory Pressure meter (CareFusion, San Diego, USA) in accordance with the protocol described by 
the American Thoracic Society and European Respiratory Society(18). This device has excellent reliability (ICC 0.83-0.90)(19).

Following successful ventilator liberation, inspiratory muscle fatigue was measured using the fatigueresistance index (FRI) previously described in ICU survivors(20). This technique, based on the Maximum Incremental Threshold Loading test(21), requires participants to breathe against 30\% resistance for 2 minutes, and MIP measures before and after the loading test are compared. FRI was also measured 1 week following successful ventilator liberation.

Participants' quality of life was measured on enrolment and completion (1 week following ventilator liberation) by research nurses blinded to group allocation. Quality of Life was measured using both the SF-36v2 tool (acute 1 week time frame) (under license QualityMetric USA) and the EQ-5D-3L tool (under license EuroQol International). The SF-36 is reliable, responsive, and has both construct and criterion validity in intensive care patients(22). The EQ-5D-3L tool has been used extensively in ICU patient followup(23) and gives a more general measure of health-related quality of life than the SF-36.

Dyspnea was measured using a Modified Borg Dyspnea scale, where dyspnea is a patient-reported categorical score out of 10 . This scale has acceptable reliability and validity in patients undergoing mechanical ventilation(24). Dyspnea was recorded both at rest (sitting comfortably in the chair or bed) and during exercise (the peak exercise activity experienced in the previous 24 hours) by research nurses blinded to group allocation, at both enrolment and study completion.

Physical function was assessed using the Acute Care Index of Function (ACIF)(25). This tool captures mental status, bed mobility, transfers and mobility, and has excellent inter-rater reliability in ICU patients $(I C C=0.94)(26)$. On enrolment, ACIF scores were completed by ICU physiotherapists prior to randomisation (thus blinded to group allocation), however follow-up ACIF scores were recorded by the ward physiotherapist who was not blinded.

Other outcomes extracted from the hospital databases included the number of training sessions (intended and completed), any requirement for reintubation, duration of mechanical ventilation, duration of pressure support ventilation, ICU length of stay, post-ICU hospital length of stay and in-hospital mortality.

\section{Data analysis}

The sample size was calculated a priori for the primary outcome measures (MIP). To detect a 10\% change in MIP with a power of $0.80,70$ participants were required (inflating group size by $15 \%$ due to anticipated mortality of $12.8 \%(12))$. In the absence of an established minimal clinically important difference in MIP in ICU patients, the $10 \%$ level was selected to facilitate comparison with previous studies of ICU survivors $(20,27)$. Raw MIP scores were normalised(28) to account for variations of MIP with age and gender. 
Paired t-tests were used to compare within group differences. Mixed linear models were used to assess the between-group difference of the changes between enrolment and follow-up measures, including age, gender, APACHE II scores and 'ventilation time prior to randomisation' as covariates. Diagnostic plots (predicted means versus Pearson's residuals) were generated to assess model assumptions. Mortality and reintubation data were analysed using Fisher's exact test. Post-ICU length of stay was analysed using a Wilcoxon rank-sum test, with exclusion of patients who died in-hospital.

Statistical significance was set as $p<0.05$. All analyses were done using R 3.6.1.

\section{Results}

The flow of participants is presented in Fig. 2. Between February 2011 and June 2019, 70 participants were recruited to participate in the study with 33 allocated to the IMT group and 37 to the control group.

The most frequent reason for exclusion from the study was low neurological status (low GCS) with inability to provide consent. Five participants were lost to follow-up in the IMT group, and ten in the control group, most commonly due to death (either during the ICU admission or following discharge on the ward). The total ICU mortality for the cohort was $10 \%$ (7/70), while the total in-hospital mortality was $18.5 \%(13 / 70)$. Where patients were lost to follow-up regarding the primary outcome measures, but for reasons other than death, secondary measures were obtained through hospital databases and telephone interview where possible.

Groups were generally comparable at baseline with participant characteristics on enrolment are presented in Table 1.

\section{Table 1}

\section{Characteristics of participants at enrolment.}




\begin{tabular}{|c|c|c|c|c|c|}
\hline \multirow[t]{2}{*}{ Characteristic } & \multicolumn{3}{|c|}{$\begin{array}{l}\text { Randomized } \\
(n=70)\end{array}$} & \multicolumn{2}{|c|}{$\begin{array}{l}\text { Lost to follow up } \\
(n=17)\end{array}$} \\
\hline & $\begin{array}{l}\text { IMT } \\
(n=33)\end{array}$ & $\begin{array}{l}\text { Control } \\
(n=37)\end{array}$ & $\begin{array}{l}\text { Total } \\
(\mathrm{n}=70)\end{array}$ & $\begin{array}{l}\text { IMT } \\
(\mathrm{n}=7)\end{array}$ & $\begin{array}{l}\text { Control } \\
(n=10)\end{array}$ \\
\hline Age $(y r)$, mean (SD) & $60(17)$ & $59(15)$ & $60(16)$ & $62(11)$ & $60(8)$ \\
\hline [Min, Max] & {$[18,82]$} & {$[19,83]$} & {$[18,83]$} & {$[45,77]$} & {$[46,71]$} \\
\hline Gender, n males (\%) & $18(55)$ & $23(62)$ & $41(59)$ & $4(57)$ & $8(80)$ \\
\hline \multicolumn{6}{|l|}{ Diagnosis, n (\%) } \\
\hline Sepsis & $5(15)$ & $5(14)$ & $10(14)$ & $3(43)$ & $3(30)$ \\
\hline Pneumonia & $1(3)$ & $6(16)$ & $7(10)$ & - & $1(10)$ \\
\hline Multitrauma & $3(9)$ & $5(14)$ & $8(11)$ & $1(14)$ & - \\
\hline Cerebrovascular Accident & $4(12)$ & - & $4(6)$ & $1(14)$ & - \\
\hline Guillain Barre Syndrome & $4(12)$ & $3(8)$ & $7(10)$ & $1(14)$ & - \\
\hline Respiratory Failure & $2(6)$ & $4(11)$ & $6(9)$ & - & $1(10)$ \\
\hline Cardiothoracic Surgery & $2(6)$ & $3(8)$ & $5(7)$ & - & $2(20)$ \\
\hline Abdominal Surgery & $4(12)$ & $1(3)$ & $5(7)$ & $1(14)$ & - \\
\hline Necrotising pancreatitis & $1(3)$ & $5(14)$ & $6(9)$ & - & $2(20)$ \\
\hline Encephalopathy / Seizures & $2(6)$ & - & $2(3)$ & - & - \\
\hline Cardiac arrest & $2(6)$ & - & $2(3)$ & - & $1(10)$ \\
\hline Other & $3(9)$ & $5(14)$ & $3(4)$ & - & - \\
\hline $\begin{array}{l}\text { APACHE II scores } \\
\text { Median [Min, Max] } \\
(25,75 \% \text { quartiles })\end{array}$ & $\begin{array}{l}19[7,40] \\
(16.25)\end{array}$ & $\begin{array}{l}18[8,34] \\
(14,24)\end{array}$ & $\begin{array}{l}19[7,40] \\
(15,24)\end{array}$ & $21[15,27]$ & $20[11,34]$ \\
\hline $\begin{array}{l}\text { Highest SOFA score } \\
\text { Median [Min, Max] } \\
(25,75 \% \text { quartiles })\end{array}$ & $\begin{array}{l}8[0,15] \\
(5,10)\end{array}$ & $\begin{array}{l}8[1,35] \\
(5,10)\end{array}$ & $\begin{array}{l}8[0,35] \\
(5,10)\end{array}$ & $9[3,15]$ & $9[2,17]$ \\
\hline
\end{tabular}

IMT = Inspiratory Muscle Training group, SD = standard deviation, CVA = cerebrovascular accident, APACHE II = acute physiology and chronic health evaluation score; SOFA = sequential organ failure assessment score 


\section{Compliance with trial protocol}

In the IMT group, the 33 participants completed a median of 8 sessions of IMT during the study (range $2-67$ ). Participants completed $71 \%$ of all intended IMT sessions (range $27 \%-100 \%)$. While $19(58 \%)$ participants completed more than $70 \%$ of the prescribed IMT sessions, $2(6 \%)$ participants completed $30 \%$ or less of the prescribed IMT sessions. The most frequent reason for lack of completion was participant refusal due to generalised fatigue, followed by confusion or drowsiness rendering them temporarily unable to participate. IMT was generally well-tolerated and no adverse effects were reported during or immediately after training in any participant. No participants in the control group inadvertently received IMT.

\section{Effect of intervention}

Changes in outcome measures within and between groups are summarized in Table E1 and Table 2. Both the IMT group and the control group significantly increased MIP scores (\% of predicted) across the study period (14.7 and $11.4 \%$ respectively)(Fig. 3a and Table 2), however the IMT group failed to demonstrate a significant increase from baseline to ventilator liberation, indicating that the majority of their improvements occurred in the final week between liberation and completion (10.1\%). There was no statistically significant difference between groups for changes in MIP from baseline to completion ( $95 \%$ Cl -7.4 to 14.0$)$.

While FRI was relatively preserved in the IMT group between ventilator liberation and study completion, the control group had a statistically significant decrease in FRI between these timepoints (-0.15) (Fig. 3b). There was no statistically significant difference between groups for changes in FRI across the study period ( $95 \% \mathrm{Cl}-0.003$ to 0.436$)$.

With regards to quality of life, the IMT group significantly increased EQ5D scores (23/100), while the control group's increase was not significant (6/100), where the minimal clinically important difference is 8. (29) The IMT group's improvement in EQ5D scores was statistically significant relative to the control group (17.2, 95\% Cl 1.3-33.0). Using the SF36 tool for quality of life, neither the mental component (MCS) nor total (SF6D) scores showed significant within-group changes; however the physical component score (PCS) significantly increased in the IMT group (6.4), while there was a non-significant decrease in the control group (-0.70). The between-group difference in change in the PCS (6.97) was statistically significant $(96 \% \mathrm{Cl} 1.96-12.00)$.

Changes in secondary outcome measures are detailed in Table E1 and Table 2. Physical function (ACIF) significantly increased in the control group only (0.14) but there was no significant difference between groups ( $95 \% \mathrm{Cl}-0.20$ to 0.08 ). Both groups showed improvements in dyspnea scores at rest and during exercise, however only the IMT group's reductions were statistically significant (-1.5 at rest, -1.9 during 
exercise). These improvements exceed the minimal clinically important difference for the Borg dyspnea scale (1 unit)(30).

\section{Table 2}

Differences within and between groups for each outcome measure comparing enrolment and completion values. 


\section{Outcome}

Differences within groups
Differences between groups across study period

(Mixed Model Analysis)

Difference between timepoints

(baseline \& completion unless

otherwise specified)

Mean (SEM)

$\begin{array}{llll}\text { IMT } & \text { Control } & \begin{array}{l}\text { Difference } \\ \text { between groups }\end{array} & \begin{array}{l}95 \% \\ \text { confidence } \\ \text { interval }\end{array}\end{array}$

MIP \% predicted

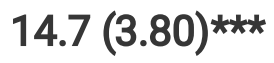

$11.4(3.83) *$

3.3

$-7.4-14.0$

Baseline to

completion

MIP \% predicted

$4.6(3.74)$

$11.0(3.91)^{*}-6.3$

$-17.1-4.4$

Baseline to

liberation

MIP \% predicted

$10.1(3.79) *$

$0.4(3.98)$

9.7

$-1.2-20.5$

Liberation to

completion

\begin{tabular}{|c|c|c|c|c|}
\hline $\begin{array}{l}\text { Fatigue resistance } \\
\text { index } / 1.00\end{array}$ & $+0.07(0.08)$ & $-0.15(0.07) *$ & 0.21 & $-.003-0.426$ \\
\hline $\begin{array}{l}\text { QOL: SF-36 PCS } \\
\text { (Physical) }\end{array}$ & 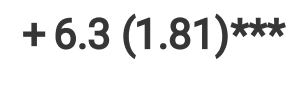 & $-0.6(1.72)$ & $6.97 *$ & $1.96-12.00$ \\
\hline $\begin{array}{l}\text { QOL: SF-36 MCS } \\
\text { (Mental) }\end{array}$ & $-1.0(3.25)$ & $+2.7(3.08)$ & -3.76 & $-12.7-5.2$ \\
\hline $\begin{array}{l}\text { QOL: SF-36 SF6D } \\
\text { (Total) }\end{array}$ & $0.046(0.0352)$ & $0.024(0.0338)$ & 0.022 & $-0.08-0.12$ \\
\hline QOL: EQ5D / 100 & 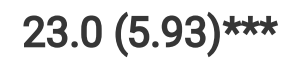 & $5.8(5.26)$ & $17.2^{*}$ & $1.3-33.0$ \\
\hline $\mathrm{ACIF} / 1.00$ & $0.08(0.053)$ & $0.14(0.050) * \star$ & -0.06 & $-0.20-0.08$ \\
\hline Dyspnea at rest /10 & $-1.5(0.67)^{*}$ & $-0.9(0.60)$ & -0.5 & $-2.33-1.28$ \\
\hline $\begin{array}{l}\text { Dyspnea during } \\
\text { exercise } / 10\end{array}$ & $-1.9(0.81) *$ & $-0.4(0.72)$ & -1.6 & $-3.77-0.55$ \\
\hline
\end{tabular}


Reintubation occurred in almost twice as many patients in the control group than the IMT group (28 vs 15 patients experienced reintubation)(Table 3), however this difference was not statistically significant.

Despite a higher median length of hospital stay in the IMT group (32 vs 21 days)(figure E1), neither postICU length of stay nor mortality were significantly different between the 2 groups. Details of patients who died during their hospital admission are included in Table E2 (online supplement).

Table 3

Comparisons between groups for ventilation, length of stay and mortality outcome measures.

\begin{tabular}{|c|c|c|c|}
\hline Outcome & $\begin{array}{l}\text { Randomized } \\
(n=70)\end{array}$ & & $\begin{array}{l}\text { Between-Group } \\
\text { Analysis }\end{array}$ \\
\hline & $\begin{array}{l}\text { IMT } \\
(n=33)\end{array}$ & $\begin{array}{l}\text { Control } \\
(n=37)\end{array}$ & \\
\hline $\begin{array}{l}\text { Duration of Mechanical Ventilation (days) } \\
\text { Median [Min, Max] } \\
(25,75 \% \text { quartiles) }\end{array}$ & $\begin{array}{l}21[7,205 \\
(14,42)\end{array}$ & $\begin{array}{l}19[8- \\
184] \\
(12,41)\end{array}$ & $p=0.57^{\#}$ \\
\hline $\begin{array}{l}\text { Duration of Pressure Support Ventilation (days) } \\
\text { Median [Min, Max] } \\
(25,75 \% \text { quartiles) }\end{array}$ & $\begin{array}{l}15[0-168] \\
(9,34)\end{array}$ & $\begin{array}{l}13[1- \\
184] \\
(8,33)\end{array}$ & $p=0.56^{\#}$ \\
\hline $\begin{array}{l}\text { Length of ICU stay (days) } \\
\text { Median [Min, Max] } \\
(25,75 \% \text { quartiles })\end{array}$ & $\begin{array}{l}30[11- \\
212] \\
(19,52)\end{array}$ & $\begin{array}{l}27[9- \\
138] \\
(15,44)\end{array}$ & $p=0.33^{\#}$ \\
\hline $\begin{array}{l}\text { Post-ICU hospital stay - hospital survivors } \\
\text { (days) } \\
\text { Median [Min, Max] } \\
(25,75 \% \text { quartiles })\end{array}$ & $\begin{array}{l}32[0-268] \\
(16,70)\end{array}$ & $\begin{array}{l}21[1- \\
277] \\
(11,52)\end{array}$ & $p=0.23^{\#}$ \\
\hline $\begin{array}{l}\text { Reintubation frequency number patients } \\
\text { reintubated } \\
\text { (Range of reintubation frequency per patient) }\end{array}$ & $\begin{array}{l}15 \\
(0-1)\end{array}$ & $\begin{array}{l}28 \\
(0-4)\end{array}$ & $\begin{array}{l}\text { OR } 0.603 \\
95 \% \text { Cl } 0.25-1.40\end{array}$ \\
\hline In-hospital mortality n (\%) & $4(12 \%)$ & $9(24 \%)$ & $\begin{array}{l}\text { OR } 0.434 \\
95 \% \text { Cl } 0.09-1.78\end{array}$ \\
\hline $\mathrm{IMT}=$ Inspiratory Muscle Training group. ICU = i & sive care un & $\mathrm{OR}=\mathrm{odd}$ & tio. \\
\hline \# Wilcoxon rank-sum test analysis. & & & \\
\hline
\end{tabular}




\section{Discussion}

In this randomized trial of mechanical threshold-based IMT commenced while ICU patients were ventilator-dependent, the main findings were that despite no difference between groups with respect to inspiratory muscle strength (MIP) or endurance (FRI), the IMT group showed significantly greater improvements in quality of life. These quality of life improvements were detected across two separate validated measures, the SF-36 (physical component score) and the EQ-5D.

The lack of improvement in MIP is somewhat surprising, given that our previous study in ICU patients, using a mechanical IMT device and high-intensity protocol, showed significant improvement in MIP within 2 weeks of training(5). However, our findings are consistent with the recent study by Moreno and colleagues(11) which found no significant difference in MIP between groups using a high-intensity protocol with a threshold device. In contrast, a small pilot study by Tonella and colleagues(31), using a tapered flow resistive electronic device, found significant improvements in both MIP and time to ventilator liberation. A possible explanation is that simple mechanical threshold devices do not allow optimised training parameters due to their limited training range $\left(9-41 \mathrm{~cm} \mathrm{H}_{2} \mathrm{O}\right)$ whereas electronic IMT devices can train patients at a broader range of intensities (i.e. from $2 \mathrm{~cm} \mathrm{H}_{2} \mathrm{O}$ to $200 \mathrm{cmH}_{2} \mathrm{O}$ ) which may be more suitable for the spectrum of ICU ventilator-dependent patients(1). Future studies should determine whether electronic IMT devices are superior to mechanical threshold devices in ventilator-dependent ICU patients in terms of recovering inspiratory muscle strength.

The improvements in quality of life, in the absence of strength or endurance improvements, are intriguing but consistent with our previous study of IMT in ICU patients(5). The fact that the physical component score of the SF-36 showed improvement indicates a perceived sense of physical progress associated with IMT. The reduction in dyspnea, unique to the IMT group in our study, would also be consistent with improvements in quality of life. In broader patients groups, dyspnea has been described as a highly complex experience, unique to the individual, encompassing physical, cognitive and emotional dimensions(32). In ventilator-dependent ICU patients, we do not yet have a clear understanding of the relationship between breathing and dyspnea associated with ventilator liberation, and its impact on quality of life. It is possible that IMT trains the psychological aspects of dyspnea even more than the physical aspects in ventilator-dependent ICU patients. Future studies should explore these links as they may be key to successful ventilator liberation.

The failure of IMT to hasten ventilator liberation in this study was disappointing, given the favourable results described in previous studies of ICU patients $(4,31,33)$; however this may be related to an inadequate training stimulus (as reflected in lack of MIP improvements), or our study could be underpowered for this outcome. In contrast to our previous study in ICU patients(5), the control group had higher in-hospital mortality than the IMT group, however this difference was not statistically significant. Based on the current data, we do not believe there should be any concerns about the mortality risk of training ICU patients with IMT. 
The strengths of this study include the randomized controlled trial design with blinded outcome assessors; the recruitment of a heterogenous sample of ICU patients of all ages and a broad range of pathologies (including those with tracheostomies); and the inclusion of patient-centred outcomes including quality of life and perception of dyspnea. Limitations include the exclusion of patients who cannot give consent, but could potentially benefit from training if sufficiently cooperative (e.g. people with brain injuries, developmental delay or delirium); the limitation of the mechanical training device (a floor of $9 \mathrm{cmH}_{2} \mathrm{O}$ and a ceiling of $41 \mathrm{cmH}_{2} \mathrm{O}$ ), which may have hampered training efficacy; and the fact that neither therapists nor patients could be blinded to the intervention. Furthermore, it is likely that this study was inadequately powered for some secondary outcomes (e.g. physical function, time to ventilator liberation, length of stay). Extrapolation of our results is also limited to intensive care units which practice minimal sedation, early rehabilitation and mobilization as this is our standard of care(34).

\section{Conclusions}

In ICU patients who have been ventilator-dependent for 7 days or longer, and are alert and able to participate in training, supervised IMT with a mechanical threshold device and a high-intensity protocol is likely to improve quality of life and dyspnea. However, inspiratory muscle strength and endurance may not improve with this training approach, and liberation from ventilation may not be accelerated.

\section{Abbreviations}

ACIF

APACHE II

$\mathrm{Cl}$

COPD

EQ-5D

FRI

GCS

ICC

ICU

IMT

MIP

QOL
Acute Care Index of Function

Acute Physiology and Chronic Health Evaluation II

Confidence Interval (95\%)

Chronic Obstructive Pulmonary Disease

EuroQuol quality of life questionnaire

Fatigue Resistance Index

Glasgow Coma Score

Intra-class correlation coefficient

Intensive Care Unit

Inspiratory Muscle Training

Maximum Inspiratory Pressure

Quality of Life 


\section{Declarations}

\section{Ethics approval and consent to participate:}

This study was approved by the Australian Capital Territory Health Human Research Ethics Committee (ETH.10.10.370) and the University of Queensland Medical Research Ethics Committee (2010001488). Participants provided their own written consent.

\section{Consent for publication:}

Not applicable(simulation equipment only in Figure 1; hands are those of researcher BB, who gives permission for this image to be used.)

\section{Availability of data and materials:}

The datasets generated and/or analysed during the current study are not publicly available due to requirements of institution as per ethical approvals, but deidentified datasets are available from the corresponding author on reasonable request.

Competing interests: The authors state they have no competing interests to declare.

\section{Funding sources:}

We gratefully acknowledge theCanberra Hospital Private Practice Fund and the Canberra Hospital Auxiliary Research Fund for supporting this study. Neither funding body had any influence over study design, analysis or manuscript development at any stage.

\section{Author contributions:}

BB had full access to all the data in the study and takes responsibility for the integrity of the data and the accuracy of the analysis. TN substantially contributed to the data analysis, and the writing of the manuscript. IAL substantially contributed to the study design, data interpretation, and the writing of the manuscript. MG, VM, KE and FvH contributed substantially to data collection, data interpretation and the writing of the manuscript. RB and JP contributed substantially to the study design, data interpretation and the writing of the manuscript. 


\section{Acknowledgements:}

The authors gratefully acknowledge the support of the physiotherapists of the Physiotherapy Department at Canberra Hospital who provided the intervention, and the Canberra Hospital ICU research nurses especially Mary Nourse and Helen Rogers. The authors thank the patients for agreeing to participate in this study.

\section{References}

1. Bissett B, Gosselink R, van Haren FMP. Respiratory Muscle Rehabilitation in Patients with Prolonged Mechanical Ventilation: A Targeted Approach. Crit Care. 2020;24(1):103.

2. Dres M, Dube BP, Mayaux J, Delemazure J, Reuter D, Brochard L, et al. Coexistence and Impact of Limb Muscle and Diaphragm Weakness at Time of Liberation from Mechanical Ventilation in Medical Intensive Care Unit Patients. Am J Respir Crit Care Med. 2017;195(1):57-66.

3. Worraphan S, Thammata A, Chittawatanarat K, Saokaew S, Kengkla K, Prasannarong M. Effects of inspiratory muscle training and early mobilisation on weaning of mechanical ventilation: a systematic review and network meta-analysis. Arch Phys Med Rehabil. 2020.

4. Vorona S, Sabatini U, Al-Maqbali S, Bertoni M, Dres M, Bissett B, et al. Inspiratory Muscle Rehabilitation in Critically III Adults: A Systematic Review and Meta-Analysis. Ann Am Thorac Soc. 2018;15(6):735-44.

5. Bissett BM, Leditschke IA, Neeman T, Boots R, Paratz J. Inspiratory muscle training to enhance recovery from mechanical ventilation: a randomised trial. Thorax. 2016;71(9):812-9.

6. Bissett B, Leditschke IA, Green M. Specific inspiratory muscle training is safe in selected patients who are ventilator-dependent: A case series. Intensive Crit Care Nurs. 2012;28(2):98-104.

7. Cader SA, Vale RG, Castro JC, Bacelar SC, Biehl C, Gomes MC, et al. Inspiratory muscle training improves maximal inspiratory pressure and may assist weaning in older intubated patients: a randomised trial. J Physiother. 2010;56(3):171-7.

8. Elbouhy $\mathrm{M}, \mathrm{AbdelHalim} \mathrm{H}$, Hashem A. Effect of respiratory muscles training in weaning of mechanically ventilated COPD patients. Egypt J Chest Dis Tuberc. 2014;63:679-87.

9. Dixit A, Prakash S. Effects of threshold inspiratory muscle training versus conventional physiotherapy on the weaning period of mechanically ventilated patients: a comparative study. International Journal of Physiotherapy Research. 2014;2(2):424-8.

10. Condessa RL, Brauner JS, Saul AL, Baptista M, Silva AC, Vieira SR. Inspiratory muscle training did not accelerate weaning from mechanical ventilation but did improve tidal volume and maximal respiratory pressures: a randomised trial. J Physiother. 2013;59(2):101-7.

11. Moreno LMS, Quiroga ICC, Luna ECW, Garcia AF. Efficacy of respiratory muscle training in weaning of mechanical ventilation in patients with mechanical ventilation for 48 hours or more: A Randomized Controlled Clinical Trial. Medicina Intensiva. 2019;43(2):79-89. 
12. Bissett BM, Leditschke IA, Paratz JD, Boots RJ. Protocol: inspiratory muscle training for promoting recovery and outcomes in ventilated patients (IMPROVe): a randomised controlled trial. BMJ Open. 2012;2(2):e000813.

13. Schulz KF, Altman DG, Moher D, Group C. CONSORT 2010 statement: updated guidelines for reporting parallel group randomised trials. BMJ. 2010;340:c332.

14. Riker RR, Picard JT, Fraser GL. Prospective evaluation of the Sedation-Agitation Scale for adult critically ill patients. Crit Care Med. 1999;27(7):1325-9.

15. Leditschke IA, Green M, Irvine J, Bissett B, Mitchell IA. What are the barriers to mobilizing intensive care patients? Cardiopulm Phys Ther J. 2012;23(1):26-9.

16. Bissett B, Leditschke IA, Green M, Marzano V, Collins S, Van Haren F. Inspiratory muscle training for intensive care patients: A multidisciplinary practical guide for clinicians. Aust Crit Care. 2018.

17. Cheah BC, Boland RA, Brodaty NE, Zoing MC, Jeffery SE, McKenzie DK, et al. INSPIRATIonALINSPIRAtory muscle training in amyotrophic lateral sclerosis. Amyotroph Lateral Scler. 2009;10(56):384-92.

18. Green M, Road J, Sieck G, Similowski T. Tests of respiratory muscle strength. ATS/ERS Statement on Respiratory Muscle Testing. Am J Respir Crit Care Med. 2002;166:528-47.

19. Dimitriadis Z, Kapreli E, Konstantinidou I, Oldham J, Strimpakos N. Test/retest reliability of maximum mouth pressure measurements with the MicroRPM in healthy volunteers. Respir Care. 2011;56(6):776-82.

20. Chang AT, Boots RJ, Brown MG, Paratz J, Hodges PW. Reduced inspiratory muscle endurance following successful weaning from prolonged mechanical ventilation. Chest. 2005;128(2):553-9.

21. Clanton T, Calverly P, Celli B. Tests of respiratory muscle endurance. ATS / ERS Statement on Respiratory Muscle Testing. Am J Respir Crit Care Med. 2002;166:559-70.

22. Hayes JA, Black NA, Jenkinson C, Young JD, Rowan KM, Daly K, et al. Outcome measures for adult critical care: a systematic review. Health Technol Assess. 2000;4(24):1-111.

23. Granja C, Morujao E, Costa-Pereira A. Quality of life in acute respiratory distress syndrome survivors may be no worst than in other ICU survivors. Intensive Care Med. 2003;29(10):1744-50.

24. Powers J, Bennett SJ. Measurement of dyspnea in patients treated with mechanical ventilation. Am J Crit Care. 1999;8(4):254-61.

25. Roach KE, Van Dillen LR. Development of an Acute Care Index of Functional status for patients with neurologic impairment. Phys Ther. 1988;68(7):1102-8.

26. Bissett B, Green M, Marzano V, Byrne S, Leditschke I, Neeman T, et al. Reliability and utility of the acute care index of function in intensive care patients: an observational study. Heart Lung. 2015; In press.

27. Chang AT, Boots RJ, Henderson R, Paratz JD, Hodges PW. Case report: inspiratory muscle training in chronic critically ill patients-a report of two cases. Physiother Res Int. 2005;10(4):222-6. 
28. Evans JA, Whitelaw WA. The assessment of maximal respiratory mouth pressures in adults. Respir Care. 2009;54(10):1348-59.

29. Zanini A, Aiello M, Adamo D, Casale S, Cherubino F, Della Patrona S, et al. Estimation of minimal clinically important difference in EQ-5D visual analog scale score after pulmonary rehabilitation in subjects with COPD. Respir Care. 2015;60(1):88-95.

30. Ries AL. Minimally clinically important difference for the UCSD Shortness of Breath Questionnaire, Borg Scale, and Visual Analog Scale. COPD. 2005;2(1):105-10.

31. Tonella RM, Ratti L, Delazari LEB, Junior CF, Da Silva PL, Herran A, et al. Inspiratory Muscle Training in the Intensive Care Unit: A New Perspective. J Clin Med Res. 2017;9(11):929-34.

32. Hayen A, Herigstad M, Pattinson KT. Understanding dyspnea as a complex individual experience. Maturitas. 2013;76(1):45-50.

33. Elkins $M$, Dentice R. Inspiratory muscle training facilitates weaning from mechanical ventilation among patients in the intensive care unit: a systematic review. J Physiother. 2015;61(3):125-34.

34. Green M, Marzano V, Leditschke I, Mitchell I, Bissett B. Mobilization of intensive care patients: a multidisciplinary practical guide for clinicians. Journal of Multidisciplinary Healthcare. 2016;9:24756.

\section{Figures}




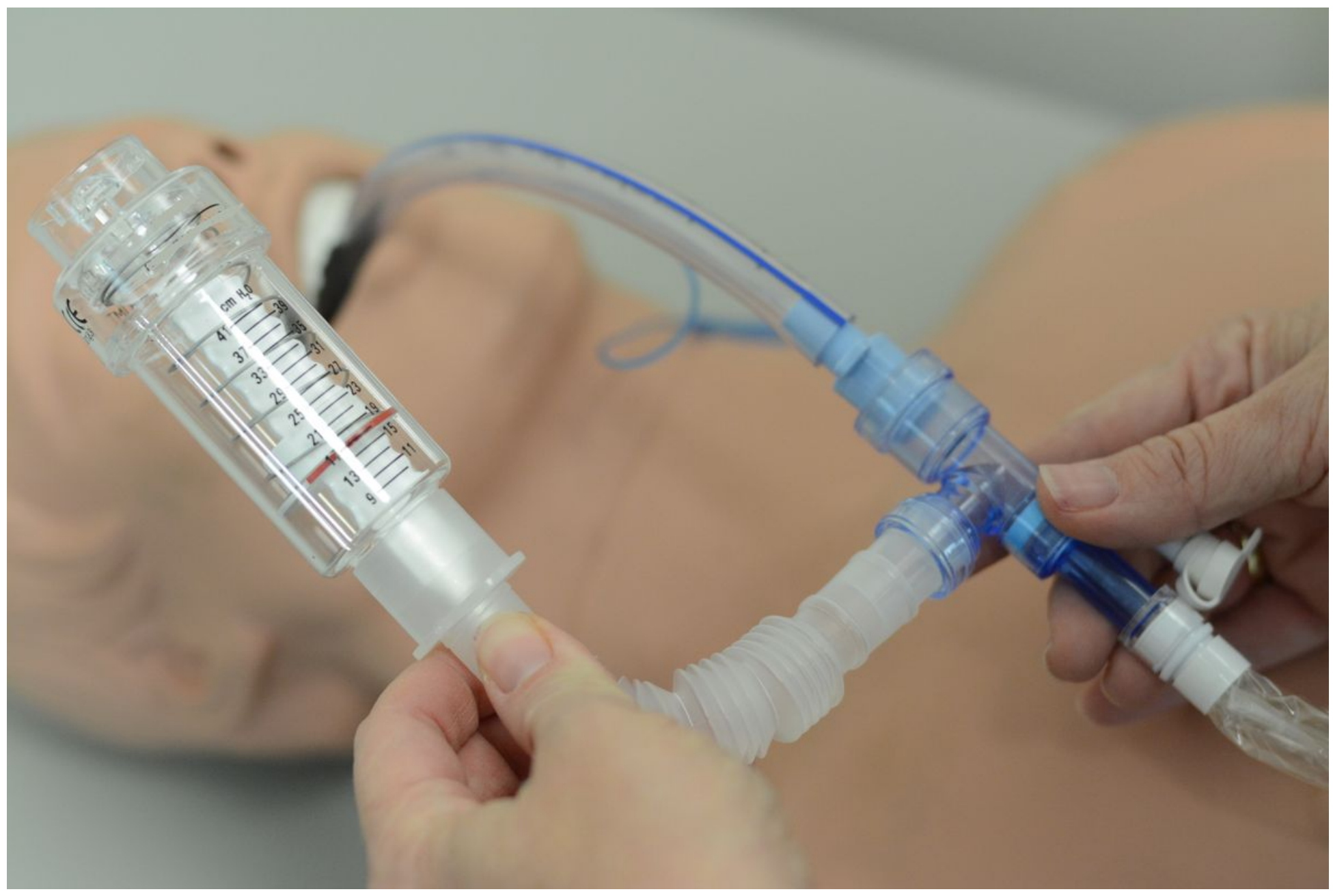

Figure 1

Attachment of threshold inspiratory muscle trainer to endotracheal tube 


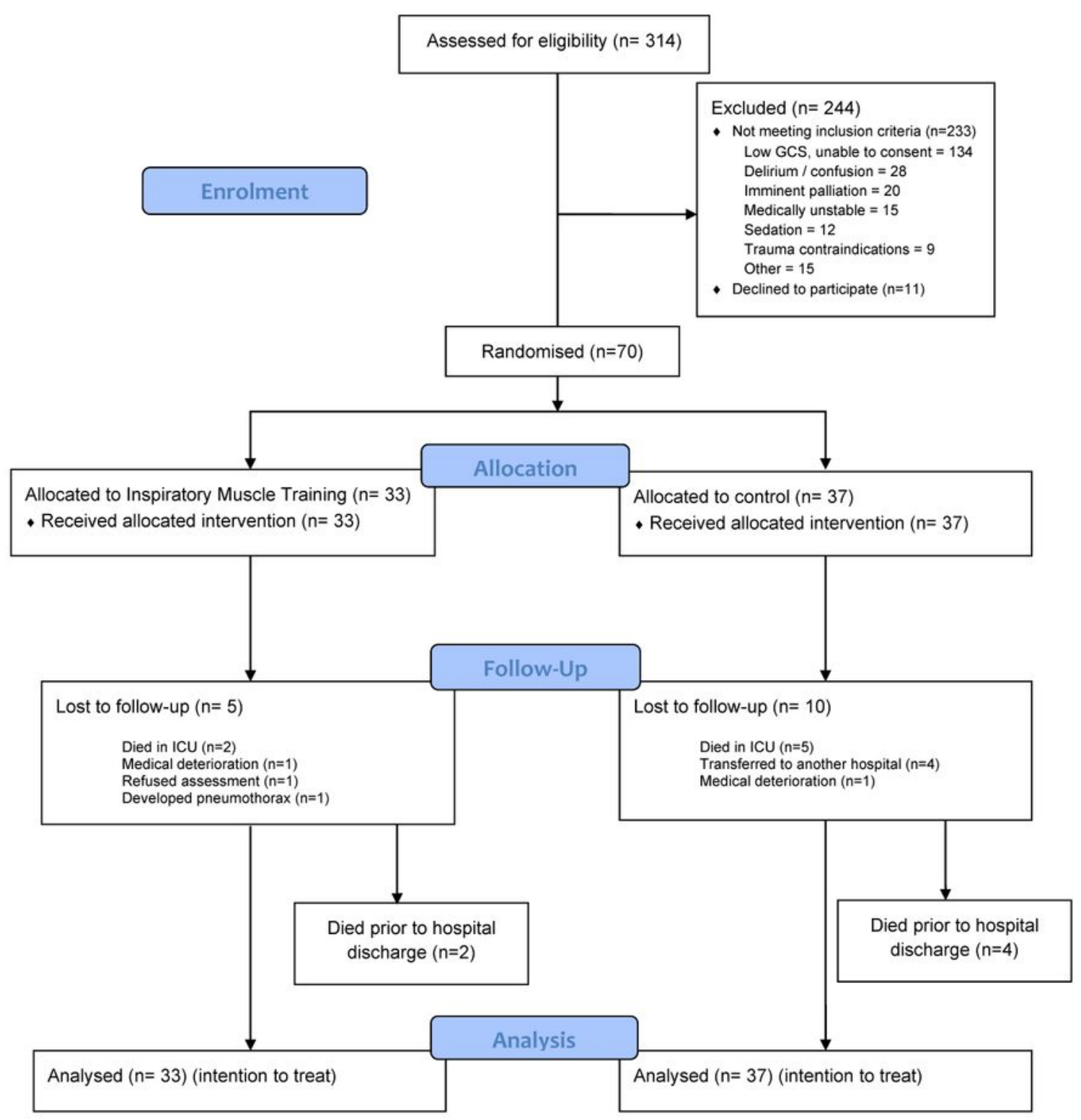

\section{Figure 2}

Flow of participants through the study 

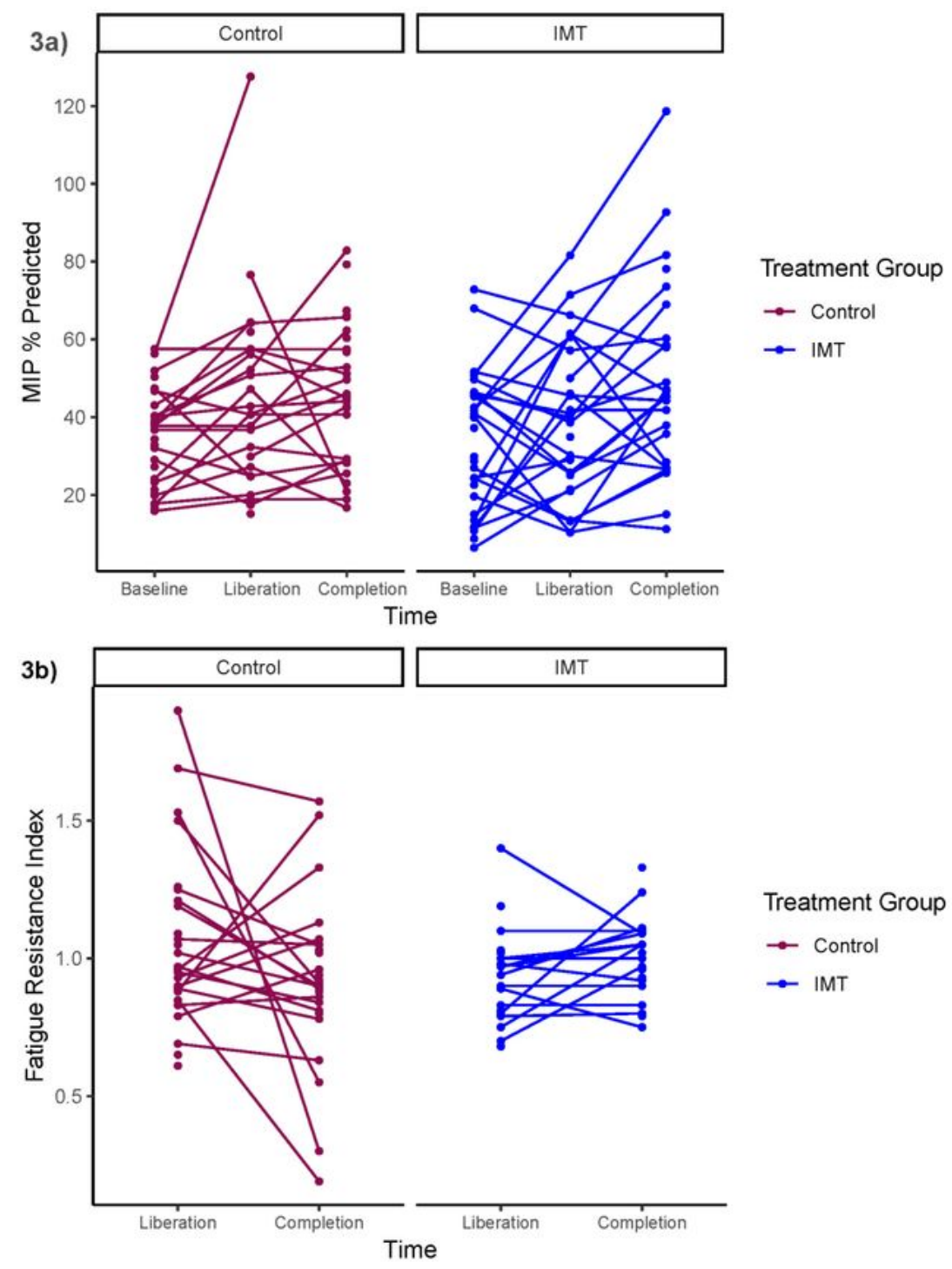

\section{Figure 3}

a) Changes in MIP (\%predicted) between baseline, ventilator liberation and study completion. b) Changes in FRI between ventilator liberation and study completion. 

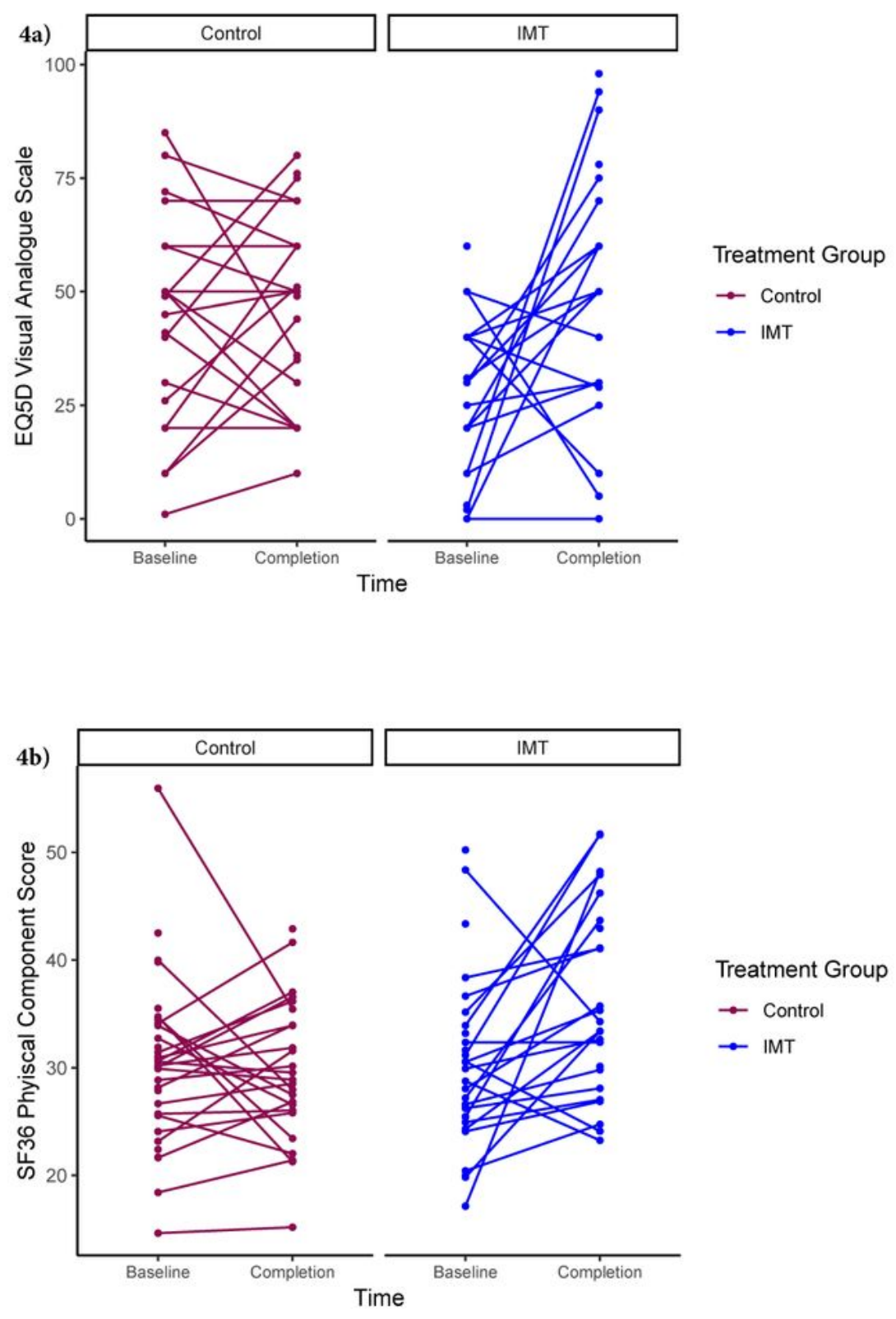

Figure 4

Changes from enrolment to completion in a) EQ5D (Visual Analogue Scale) scores and b) SF36 Physical Component Score changes

\section{Supplementary Files}


This is a list of supplementary files associated with this preprint. Click to download.

- 2021RCTAdditionalFile1.pdf

- RCT2021CONSORT2010ChecklistMSWord.doc 